\title{
Lower Bounds for Off-Line Range Searching*
}

\author{
B. Chazelle \\ Department of Computer Science, Princeton University, \\ Princeton, NJ 08544, USA \\ chazelle@cs.princeton.edu
}

\begin{abstract}
This paper proves three lower bounds for variants of the following rangesearching problem: Given $n$ weighted points in $\mathbf{R}^{d}$ and $n$ axis-parallel boxes, compute the sum of the weights within each box: (1) if both additions and subtractions are allowed, we prove that $\Omega(n \log \log n)$ is a lower bound on the number of arithmetic operations; (2) if subtractions are disallowed the lower bound becomes $\Omega\left(n(\log n / \log \log n)^{d-1}\right)$, which is nearly optimal; (3) finally, for the case where boxes are replaced by simplices, we establish a quasi-optimal lower bound of $\Omega\left(n^{2-2 /(d+1)}\right) / \operatorname{polylog}(n)$.
\end{abstract}

\section{Introduction}

We establish three lower bounds for problems of the following kind: Given $n$ weighted points in $\mathbf{R}^{d}$ and $n$ axis-parallel boxes, compute the sum of the weights within each box. The dimension $d$ is arbitrary but fixed. Problems of this sort have been extensively studied: see [5], [8], [13], [14], [16], and [19] for surveys or general introductions to the subject of range searching. We prove that:

- If both additions and subtractions are allowed, then the problem requires $\Omega(n \log \log n)$ arithmetic operations. This is the first general result for the group model. Note, however, that it falls short of the best known upper bound of $O(n \log n)$. The proof uses the spectral method which we introduced in [6]. This reduces the problem to that of finding a set system with incidence matrix $A$ such that the eigenvalues of $A^{\top} A$ are large. We do this nonconstructively by using a mixture of algebraic and probabilistic arguments. The key ingredient of the proof is a

* A preliminary version of this paper appeared in Proc. 27th Ann. ACM Symp. on Theory of Computing, May 1995, pp. 733-740. This work was supported in part by NSF Grant CCR-93-01254 and the Geometry Center. University of Minnesota, an STC funded by NSF, DOE, and Minnesota Technology, Inc. 
discrete version of Roth's method of orthogonal functions. This is a powerful technique from discrepancy theory, which we hope will find further use in complexity theory.

- If subtractions are disallowed (the semigroup model), then a much stronger lower bound can be established, i.e., $\Omega\left(n(\log n / \log \log n)^{d-1}\right)$, which is nearly optimal. The semigroup model corresponds to the monotone arithmetic circuit complexity of the problem, so it should be expected that lower bounds are easier to prove. Actually, the proof is surprisingly simple: it involves little more than the Chinese Remainder Theorem and basic properties of Halton-Hammersley sequences. The on-line version of the problem was treated in [4] and [10] and required fairly complicated arguments. The off-line case was open.

- Our last result concerns the same problem as above, but with simplices replacing boxes. Again, a quasi-optimal lower bound can be established in the semigroup model, i.e., $\Omega\left(n^{2-2 /(d+1)}(\log n)^{-5 / 2}\right)$. A practical observation is that when $d$ is large the bound is basically quadratic, which shows that the naive algorithm (checking which point belongs to which simplex, one pair at a time) is the method of choice. The proof makes use of recent results on Heilbronn's problem [3] and techniques from [7]. Again, the on-line version of the problem has been (almost completely) solved [3], [11], [18], while the off-line case was open. See also [9] for related results on Hopcroft's problem in two dimensions.

\section{Lower Bounds in the Group Model}

Given $n$ weighted points in the plane, with weights chosen in an Abelian group $(G,+)$, and $n$ axis-parallel boxes, we consider the problem of computing the sum of the weights of the points within each box. Obviously this is the same as computing $A x$, where $A$ is the incidence matrix of the associated set system and $x$ is the vector of weights.

In the group model a circuit (or straight-line program) encodes the map $A$ and is required to compute $A x$ for any $x \in G^{n}$, where $(G,+)$ is an arbitrary Abelian group. There are two types of gates: A regular gate takes a pair $(a, b)$ as input and it outputs $a+b$ or $a-b$. A help gate outputs $f(a, b)$, where $f$ is any function from $G^{2}$ to $G$. The motivation behind the use of help gates is that often the group $G$ can be embedded into a more complex structure, say, a ring or a field, and other operations might be possible. The use of help gates leaves open that possibility without restricting the generality of the model.

For the purpose of the proof we assume that $G$ is the additive group of real numbers. Let $\lambda_{1} \geq \cdots \geq \lambda_{n}$ be the eigenvalues of $A^{\top} A$. The spectral lemma of [6] states that any circuit for computing $A x$ is of size $\Omega\left(\max _{k}(k-2 h) \log \lambda_{k}\right)$, where $h$ is the number of help gates. (This generalizes Morgenstern's classical result [15].) Thus we construct a set of $n$ points and a set of $n$ axis-parallel boxes such that any eigenvalue of $A^{\top} A$ of rank slightly less than $n / 4$ is at least $(\log n)^{\Omega(1)}$. This immediately implies that computing $A x$ requires a circuit of size $\Omega(n \log \log n)$, even in the presence of up to roughly $n / 8$ help gates.

First, we build a large $N \times n$ set system $B$, from which we extract an $n \times n$ set system $A$ that satisfies the lower bound. To construct $B$ we use a set $P$ of $n$ points given by a (well-chosen) two-dimensional Halton-Hammersley subsequence; for boxes we 
take the southwest quadrants cornered at the $N$ vertices of a very fine square grid. Let $\mu_{1} \geq \cdots \geq \mu_{n}$ be the eigenvalues of $B^{\top} B$. (In the following we make no distinction between a set system and its incidence matrix.) We go through the following sequence, from which the lower bound follows by application of the spectral lemma: ${ }^{1}$

- Step 1. Show that $\mu_{k} \gg(n-k+1) N(\log n) / n^{2}$ (Lemma 2.3). By the CourantFischer characterization of eigenvalues, this entails estimating the minmax value of the Rayleigh quotient $\|B x\|_{2}^{2} /\|x\|_{2}^{2}$ over all $(n-k+1)$-dimensional subspaces. By using Roth's method of orthogonal functions [2], [17] we derive a lower bound on the hybrid ratio $\|B x\|_{2} /\|x\|_{1}$, for any $x \neq 0$ (Lemma 2.2). Unfortunately, we need the $L^{2}$ norm in the denominator. The standard inequalities relating the $L^{1}$ and $L^{2}$ norms are too crude for our purposes, and we need a probabilistic argument to produce the desired lower bound (Lemma 2.3).

- Step 2. Prove the existence of an $n \times n$ submatrix $A$ of $B$ such that $\operatorname{det} A^{\top} A=$ $(\log n)^{n-o(n)}\left(\right.$ Lemma 2.4). Step 1 yields a lower bound on $\operatorname{det} B^{\top} B=\prod_{k} \mu_{k}$. The Binet-Cauchy formula leads to the (nonconstructive) existence of $A$.

- Step 3. Show that the $k$ th largest eigenvalue $\lambda_{k}$ of $A^{\top} A$ is at least $(\log n)^{\Omega(1)}$, for any $k$ up to roughly $n / 4$ (Lemma 2.5). Since $\operatorname{det} A^{\top} A=\prod_{k} \lambda_{k}$, such a bound follows from the previous step, provided that we can bound the low-ranked eigenvalues from above. This is done by exhibiting large enough invariant subspaces within which the spectral norm of the map $A^{\top} A$ is low.

Remark. Step 3 makes use (of all things!) of data-structuring techniques for range searching. It is ironic that proving a lower bound on the complexity of range searching should require the use of data structures. However, of course, one consequence of this work is that a low spectrum is a precondition for the existence of efficient data structures for linear maps, and hence for range searching. So, with hindsight it is not all that surprising.

Theorem 2.1. Range searching with respect to $n$ points and $n$ axis-parallel boxes requires $\Omega(n \log n \log n)$ group operations in the worst case. This remains true even in the presence of $n / 8-\varepsilon n$ help gates, for any fixed $\varepsilon>0$.

The theorem shows that up to $n / 8$ help gates cannot help. On the other hand, it is easy to see that over the reals the problem can be solved entirely with only $2 n-1$ help gates: the circuit is a tree of help gates whose leaves are the $x_{i}$ 's and whose root "collects" the vector $\left(x_{1}, \ldots, x_{n}\right)$ and encodes it as real. Then, with another $n$ help gates, we can distribute the correct $n$ outputs: the total number of help gates is $2 n-1$.

It should also be noted that without help gates the problem is easily solved in $O(n \log n)$ time on a RAM. The lower bound holds in any dimension higher than one. The obvious open question is whether $\Theta(n \log \log n)$ is the right bound. The spectral method seems unlikely to provide an answer to this question. For example, it would be expected that the dimension of the ambient space plays a role, something which the method seems to rule out.

\footnotetext{
' We use the notation $\gg$ or $\ll$ to denote inequality up to a constant multiplicative factor.
} 
Proof of Theorem 2.1. Let $m$ be a large power of two and let $n=m / 4$. The $n$-point set $P$ is a subset of the classical bit-reversal $m$-point set:

$$
Q=\left\{\left(\frac{1}{2 m}+c(k), \frac{1}{2 m}+\frac{k}{m}\right): 0 \leq k<m\right\},
$$

where $c(k)=\sum_{i \geq 0} b(k, i) / 2^{i+1}$ and $\{b(k, i)\}$ is the binary expression for $k$, i.e., $k=$ $\sum_{i \geq 0} b(k, i) 2^{i}$. For any $1 \leq k \leq \log n$, let $X_{k}$ be the grid obtained by dividing $[0,1]^{2}$ into $m$ axis-parallel rectangles of size $2^{-k} \times\left(2^{k} / m\right)$. Each cell $\sigma$ of $X_{k}$ is a rectangle of area $1 / m$ that contains exactly one point $q$ of $Q$. We say that $q$ is well-centered for $X_{k}$ if it lies near the center $c_{\sigma}$ of $\sigma$; specifically, within the box $\left(\sigma+c_{\sigma}\right) / 2$. A simple inductive proof shows that at least half the points of $Q$ are well-centered for $X_{k}$. (Intuitively, for a random choice of $\sigma$ in $X_{k}$ its unique point is equally likely to lie in any of its partitioning square cells.) It follows that at least $m / 4$ points of $Q$ are each well-centered for at least $(\log n) / 3$ grids $X_{k}$. We define $P$ to consist of these $m / 4$ points.

Consider the $(\sqrt{N}-1) \times(\sqrt{N}-1)$ square grid $\mathcal{G}$ covering $[0,1]^{2}$, where $N=$ $\left(m^{2}+1\right)^{2}$. Each row of the $N \times n$ matrix $B$ is the characteristic vector of the subset of $P$ lying in the southwest quadrant cornered at a distinct grid point; in other words, for each grid point $(x, y)$ there is a distinct row in $B$ corresponding to the quadrant $(-\infty, x] \times(-\infty, y]$. Note that the $N$ rows are not all distinct. Next, we show that the set system $B$ has a high spectrum. We do this in two steps: we lower-bound successively the $L^{2}$ norm of $B x$ and the eigenvalues of $B^{\top} B$.

Lemma 2.2. For any $x \in \mathbf{R}^{n}$,

$$
\|B x\|_{2} \gg \frac{1}{n} \sqrt{N \log n}\|x\|_{1} .
$$

Proof. Fix $x=\left(x_{1}, \ldots, x_{n}\right) \in \mathbf{R}^{n}$ : each $x_{i}$ corresponds to a distinct point of $P$; for convenience we use the same notation $x_{i}$ to refer to a point and to its weight (which one is meant being obvious from context). Without loss of generality, we can assume that

$$
\|x\|_{1} \leq 2 \sum_{x_{i}>0} x_{i}
$$

Our approach is a discretized variant of Roth's method of orthogonal functions [2], [17]. Given $1 \leq k \leq \log n$, we say that a cell $\sigma$ of $X_{k}$ is $k$-good if it contains a well-centered point $x_{i}$ and $x_{i}>0$. We assign a weight to each grid point $q$ of $\mathcal{G}$ as follows: Let $\sigma$ be any cell of $X_{k}$ that contains $q$ :

- If $\sigma$ is not uniquely defined (because $q$ lies on its boundary) or if $\sigma$ is not $k$-good, then assign $q$ a weight of 0 .

- Else, subdivide $\sigma$ into four equal-size quadrants (similar to $\sigma$ ): Assign $q$ a weight of 1 if it lies in the interior of the northeast or southwest quadrant; assign a weight of -1 if it lies in the interior of the northwest or southeast quadrant. If $q$ lies elsewhere, assign it a weight of 0 .

One might recognize in the weight assignment a modification of the standard twodimensional Rademacher function. Let $g_{k} \in \mathbf{R}^{N}$ be the column vector of weights (with 
coordinates in the same order as the corresponding rows of $B$ ). It is easily checked that the $\log n$ vectors $g_{k}$ are orthogonal. Let $G$ be the matrix whose columns are the $g_{k}$ 's and let $u$ be the column vector of $\mathbf{R}^{\log n}$ whose coordinates are all ones. It follows that

$$
\|G u\|_{2}^{2}=\sum_{k=1}^{\log n}\left\|g_{k}\right\|_{2}^{2} \leq N \log n .
$$

We easily show that by summing separately over each $k$-good cell $\sigma$ we obtain

$$
g_{k}^{\top} B x \gg \frac{N}{n} \sum_{i}\left\{x_{i} \in k \text {-good cell of } X_{k}\right\} .
$$

To see why, when summing up the weighted coordinates of $B x$ over each $k$-good cell, regroup each point with its three symmetric translates (one in each subquadrant) and apply the inclusion-exclusion formula. Finally, use the well-centeredness to argue for the presence of the factor $N / n$. We omit the details, which are straightforward. Since each $x_{i}>0$ is well-centered for at least $(\log n) / 3$ grids, then, by (1),

$$
(G u)^{\top} B x \gg \frac{N \log n}{n}\|x\|_{1},
$$

and, by Cauchy-Schwarz,

$$
\frac{N \log n}{n}\|x\|_{1} \ll(G u)^{\top} B x \leq\|G u\|_{2} \cdot\|B x\|_{2} \leq \sqrt{N \log n}\|B x\|_{2} .
$$

We are now ready to complete the first step of our lower-bound proof and estimate the eigenvalues of $B^{\top} B$ from below. Let $\mu_{1} \geq \cdots \geq \mu_{n} \geq 0$ be the eigenvalues of $B^{\top} B$.

Lemma 2.3. For any $1 \leq k \leq n$, the $k$ th largest eigenvalue of $B^{\top} B$ satisfies

$$
\mu_{k} \gg \frac{(n-k+1) N \log n}{n^{2}} .
$$

Proof. Let $\left\{v_{i}\right\}$ be an orthonormal eigenbasis for $B^{\top} B$, where $v_{i}$ is associated with $\mu_{i}$, and let $F$ be the invariant subspace spanned by $v_{k}, \ldots, v_{n}$. By the Courant-Fischer theorem we know that

$$
\mu_{k}=\max _{0 \neq x \in F} \frac{\|B x\|_{2}^{2}}{\|x\|_{2}^{2}} .
$$

The difficulty is that in Lemma 2.2 the $L^{2}$ norm of $B x$ is bounded in terms of the $L^{1}$ norm of $x$. On the other hand, the inequality $\|x\|_{1} \geq\|x\|_{2}$ is too weak for our purposes, so we argue (probabilistically) that the subspace $F$ is "big"enough so that it contains vectors whose $L^{1}$ and $L^{2}$ norms deviate from each other substantially.

Let $\xi$ be the column vector obtained by expressing $x$ in the basis $\left\{v_{i}\right\}$ : we have $\xi=Q x$, where $Q=\left(q_{i j}\right)$ is the orthogonal matrix whose rows are the eigenvectors $v_{i}$. Let $R=\left(r_{i j}\right)$ be the matrix obtained by replacing each of the first $k-1$ rows of $Q$ 
by a row of zeros. Now let $y=\left(y_{1}, \ldots, y_{n}\right)$ be a random vector chosen uniformly in $\{-1,1\}^{n}$.

$$
\begin{aligned}
\mathbf{E}\|R y\|_{2}^{2} & =\sum_{i=1}^{n} \mathbf{E}\left(\sum_{j=1}^{n} r_{i j} y_{j}\right)^{2} \\
& =\sum_{i \geq k} \sum_{j=1}^{n} q_{i j}^{2} \mathbf{E} y_{j}^{2}+\sum_{i \geq k} \sum_{j \neq j^{\prime}} q_{i j} q_{i j^{\prime}} \mathbf{E} y_{j} y_{j^{\prime}} \\
& =\sum_{i \geq k} \sum_{j=1}^{n} q_{i j}^{2}=n-k+1 .
\end{aligned}
$$

This implies the existence of a vector $y \in\{-1,1\}^{n}$ such that $\|R y\|_{2}^{2} \geq n-k+1$, and therefore the $(n-k)$-flat defined by the equations

$$
\begin{cases}\xi_{i}=0 & (1 \leq i \leq k) \\ (Q y)^{\top} \xi=\sqrt{n-k+1}\end{cases}
$$

cuts the unit-radius ball centered at the origin. Let $x$ be a point of the intersection. Since $\xi=Q x$,

$$
\|x\|_{1} \geq y^{\top} x=(Q y)^{\top} \xi=\sqrt{n-k+1} .
$$

Applying Lemma 2.2, we derive

$$
\mu_{k} \geq \sum_{i=1}^{n} \mu_{i} \xi_{i}^{2}=\|B x\|_{2}^{2} \gg \frac{N \log n}{n^{2}}\|x\|_{1}^{2} \geq \frac{(n-k+1) N \log n}{n^{2}} .
$$

The determinant of $B^{\top} B$ is the product of the eigenvalues, therefore

$$
\operatorname{det} B^{\top} B \gg \Omega\left(\frac{N \log n}{n^{2}}\right)^{n} n !
$$

It is now easy to exhibit a hard set system $A$ for orthogonal range searching and complete the second step of the lower-bound proof.

Lemma 2.4. There exists an $n \times n$ submatrix $A$ of $B$ such that

$$
\operatorname{det} A^{\top} A=(\log n)^{n-o(n)} \text {. }
$$

Proof. By the Binet-Cauchy formula, ${ }^{2}$

$$
\operatorname{det} B^{\top} B=\sum_{1 \leq j_{1}<\cdots<j_{n} \leq N}\left|\operatorname{det} B\left(\begin{array}{cccc}
j_{1} & j_{2} & \cdots & j_{n} \\
1 & 2 & \cdots & n
\end{array}\right)\right|^{2} .
$$

\footnotetext{
2 The notation refers to the matrix obtained by picking the rows indexed $j_{1}, \ldots, j_{n}$ in $B$.
} 
Therefore by (2) there exists an $n \times n$ submatrix $A$ of $B$ such that

$$
\begin{aligned}
\operatorname{det} A^{\top} A & =\left|\operatorname{det} B\left(\begin{array}{cccc}
j_{1} & j_{2} & \cdots & j_{n} \\
1 & 2 & \cdots & n
\end{array}\right)\right|^{2} \\
& \geq\left(\begin{array}{c}
N \\
n
\end{array}\right)^{-1} \operatorname{det} B^{\top} B \\
& =\Omega(1)^{n}\left(\frac{n}{e N}\right)^{n}\left(\frac{n}{e}\right)^{n}\left(\frac{N \log n}{n^{2}}\right)^{n} \geq(\log n)^{n-o(n)}
\end{aligned}
$$

We can now move on to the last step of the lower-bound proof. Let $\lambda_{1} \geq \cdots \geq \lambda_{n} \geq 0$ be the eigenvalues of $A^{\top} A$.

Lemma 2.5. For any fixed $\varepsilon>0$ and any $k \leq n / 4-\varepsilon n$, we have $\lambda_{k}=(\log n)^{\Omega(1)}$.

Proof. The intuition is this: write down some linear constraints which, if satisfied, allow us to express the map $A$ by a matrix with only few ones. Then, by using standard matrix norm inequalities, argue that within the subspace satisfying the constraints, $\|A x\|_{2} /\|x\|_{2}$ is always small. This implies an upper bound on the eigenvalues, which allows us to conclude. We flesh out these ideas below.

Place the points $P$ in bijection with the leaves of a complete binary tree (from left to right): each node $v$ of the tree is associated with the vertically sorted list $N_{v}$ of the points stored at the leaves at or below $v$. This is a classical range-tree construction [14]. Any set specified by a row of $A$ can be partitioned into fewer than $v=\log n+1$ subsets: each subset is a prefix of a list $N_{v}$ and all the relevant lists $N_{v}$ are on different levels of the tree. Thus, we can create $v$ systems, one for each level, such that their $n \times n$ incidence matrices $A_{1}, \ldots, A_{\nu}$ satisfy $A=\sum_{i} A_{i}$.

For every level $i$, perform the following operations. First, check whether some of the rows in $A_{i}$ are identical: $\rho$ identical rows all correspond to the same prefix in some $N_{v}$. Take the last element in the prefix (i.e., the point with the highest $y$-coordinate) and duplicate it $\rho-1$ times within the list $N_{v}$. Note that the total size of the augmented lists at level $i$ is at most $2 n$. Next, consider each list $N_{v}$ (after the previous preprocessing) and subdivide it into contiguous lists of $r$ (or fewer) points; $r$ is a parameter to be specified later.

To summarize, at each level we have a collection of at most $2 n / r$ lists of size exactly $r$ along with a number of other lists of size less than $r$. We can now remove all the duplicates, as their presence was needed only to calibrate the size of the lists. (Note that in the process some lists become empty.) Any subset specified by a row of $A_{i}$ can be written as a union of full lists and a remainder set of size less than $r$. Thus, if $H$ is the matrix whose rows are the characteristic vectors of each full list (over all $A_{i}$ ), then the restriction of $A_{i}$ (viewed as a linear transformation) to $\operatorname{Ker} H$ can be expressed by an $n \times n$ matrix $C_{i}$, whose rows each have fewer than $r$ ones. Because of the earlier duplication of points in the lists, note that similarly no column of $C_{i}$ can have more than $r$ ones. The rank of $H$ does not exceed its number of rows, which is at most $2 v n / r$, 
therefore

$$
\text { codim Ker } H \leq \frac{2 v n}{r} \text {. }
$$

It is a standard result in matrix theory [12] that the spectral norm of a matrix $M$ satisfies

$$
\|M\|_{s}^{2} \leq\left(\max _{i} \sum_{j}\left|m_{i j}\right|\right)\left(\max _{j} \sum_{i}\left|m_{i j}\right|\right),
$$

and, therefore, $\left\|C_{i}\right\|_{s}^{2} \leq r^{2}$. Using the fact that

$$
\left\|C_{i} x\right\|_{2} \leq\left\|C_{i}\right\|_{s} \cdot\|x\|_{2} \leq r\|x\|_{2},
$$

we find that, for any $x \in \operatorname{Ker} H$, by the triangular inequality,

$$
\|A x\|_{2} \leq \sum_{i=1}^{\nu}\left\|A_{i} x\right\|_{2}=\sum_{i=1}^{\nu}\left\|C_{i} x\right\|_{2} \leq r \nu\left\|_{x}\right\|_{2} .
$$

By the Courant-Fischer characterization of eigenvalues, we know that

$$
\lambda_{j}=\min _{F} \max _{0 \neq x \in F} \frac{\|A x\|_{2}^{2}}{\|x\|_{2}^{2}},
$$

where the minimum is taken over all subspaces of dimension $n-j+1$. Fix $j$ such that $v \leq j \leq n$; by (3), setting $r=\lceil 2 v n /(j-1)\rceil$ makes the dimension of Ker $H$ exceed $n-j$, therefore, for $n$ large enough,

$$
\lambda_{j} \leq \max _{0 \neq x \in \text { Ker } H} \frac{\|A x\|_{2}^{2}}{\|x\|_{2}^{2}} \leq r^{2} v^{2} \leq 5\left(\frac{n \log ^{2} n}{j}\right)^{2} .
$$

Note that this inequality remains valid if $1 \leq j<v$, because of the trivial upper bound, $\lambda_{j} \leq n^{2}$. By Lemma 2.4 ,

$$
\begin{aligned}
(n-k+1) \log \lambda_{k} & \geq \log \operatorname{det} A^{\top} A-\sum_{j=1}^{k-1} \log \lambda_{j} \\
& \geq(n-o(n)) \log \log n-k(O(1)+2 \log n-2 \log k+4 \log \log n) .
\end{aligned}
$$

Choosing $k=n / 4-\varepsilon n$, for any fixed $\varepsilon>0$, gives $\lambda_{k}=(\log n)^{\Omega(1)}$.

The lower bound for range searching follows directly from the spectral lemma, which proves Theorem 2.1.

\section{The Semigroup Model}

In the semigroup version of range searching, subtractions are not allowed (or not defined). In other words, the model of computation is a straight-line program or circuit: each (charged) step is the form

$$
z \leftarrow x+y
$$


where $x$ and $y$ are previously computed variables or input weights. It is possible to weaken the model slightly (and, hence, strengthen our results) by relaxing the assumption that the program should work for all commutative semigroups. It suffices to require that it should work for at least one faithful semigroup. This is a semigroup over which any two identically equal linear forms must have the same variables (though not necessarily the same coefficients)—see [3], [4], and [18] for formal definitions.

Theorem 3.1. Range searching with respect to $n$ points and $n$ axis-parallel boxes in $\mathbf{R}^{d}$ requires on the order of $n(\log n / \log \log n)^{d-1}$ semigroup operations.

Theorem 3.2. Range searching with respect to $n$ points and $n$ simplices in $\mathbf{R}^{d}$ requires on the order of $n^{2-2 /(d+1)}(\log n)^{-5 / 2}$ semigroup operations.

Complicated arguments were used in [3] and [4] to treat the on-line cases. As it turns out, the off-line case is considerably easier. The bound of Theorem 3.1 is fairly close to the known upper bound of $n(\log n)^{d-1} \alpha(n)$ [7]. The bound of Theorem 3.2 is within a polylogarithmic factor of the best current upper bound of $n^{2-2 /(d+1)}(\log n)^{o(1)}$ [13].

The proofs of both theorems are based on a simple graph-theoretical lemma. Let $A=\left(a_{i j}\right)$ denote the $n \times n$ incidence matrix of a range-searching problem. Suppose that $A$ has no $p \times q$ submatrix of ones. An equivalent formulation is to say that the corresponding bipartite graph has no $(p, q)$ complete bipartite subgraph.

Lemma 3.3. If $A$ is an $n \times n$ incidence matrix with no $p \times q$ submatrix of ones, then the complexity of computing Ax over a semigroup is at least on the order of

$$
\frac{1}{p q}\left(\sum_{i, j} a_{i j}\right)-\frac{n}{p}
$$

Proof. Every gate of the circuit adds two linear forms together: we say that the gate is heavy if the linear form it outputs, $\sum_{j} \alpha_{j} x_{j}\left(\alpha_{j} \in \mathbf{N}^{+}\right)$, involves $q$ variables $x_{j}$ or more. Given a row $i$, let $S_{i}=\sum_{j} a_{i j}$. Because of faithfulness, the output gate $g$ computing the form $\sum_{j} a_{i j} x_{j}$ is connected to $S_{i}$ input variables $x_{i_{1}}, x_{i_{2}}$, etc. Consider a subtree $T_{i}$ of the circuit graph with $g$ as its root and $x_{i_{1}}, x_{i_{2}}, \ldots$ at its leaves. Note that the maximal subtrees of $T_{i}$ with at most $q$ leaves (i.e., those whose root's parent has more than $q$ descending leaves) are disjoint. Each such subtree has one fewer two-child nodes than leaves. There are at least $S_{i} / q$ such subtrees, so they account for at most $S_{i}-S_{i} / q$ two-child nodes. This shows that at least $S_{i} / q-1$ internal nodes of $T_{i}$ correspond to heavy gates. By faithfulness again, no heavy gate can provide a node for $p$ trees $T_{i}$. Indeed, this would create a $p \times q$ submatrix of ones in $A$. The lower bound follows immediately.

Proof of Theorem 3.1. The set of input points is obtained from a Halton-Hammersley sequence [2]. Let $p_{1}<p_{2}<\cdots<p_{d-1}$ be consecutive primes. Any integer $m$ has a unique decomposition in base $p_{k}: m=\sum_{i \geq 0} b_{k}(m, i) p_{k}^{i}$. We define the function

$$
x_{k}(m)=\sum_{i \geq 0} \frac{b_{k}(m, i)}{p_{k}^{i+1}} .
$$


This allows us to construct the input point set

$$
P=\left\{\left(x_{1}(m), \ldots, x_{d-1}(m), \frac{m}{n}\right): 0 \leq m<n\right\} .
$$

Any interval of the form $\left[M / p_{k}^{j},(M+1) / p_{k}^{j}\right)$, where $M$ is a nonnegative integer, is said to be of type $(k, j)$. A box $B$ is special if it is of the form $I_{1} \times \cdots \times I_{d}$, where

- $B \subseteq[0,1]^{d}$;

- $I_{1}, \ldots, I_{d-1}$ are intervals of type $\left(1, j_{1}\right), \ldots,\left(d-1, j_{d-1}\right)$, respectively, for some integers $j_{1}, \ldots, j_{d-1} \geq 0$

- $I_{d}$ is of the form $\left[M p_{1} Q / n,(M+1) p_{1} Q / n\right)$, where $Q=p_{1}^{j_{1}} \cdots p_{d-1}^{j_{d-1}}$ and $M$ is an integer.

We motivate this definition. First, observe that knowing which $(k, j)$-interval contains $x_{k}(m)$ amounts to the knowledge of the digits $b_{k}(m, 0), \ldots, b_{k}(m, j-1)$. Thus, if we know that the point of $P$ indexed by $m$ lies in the box $I_{1} \times \cdots \times I_{d-1} \times[0,1]$, then we know the residues classes of $m$ modulo $p_{1}^{j_{1}}, \ldots, p_{d-1}^{j_{d-1}}$, respectively. By the Chinese Remainder Theorem, this implies that we know $m$ modulo $Q$. It follows at once that each of the boxes $I_{1} \times \cdots \times I_{d-1} \times[l Q / n,(l+1) Q / n)$ contains at most one point of $P$. Actually, any special box $B$ contains exactly $p_{1}$ points of $P$. The number $N$ of such boxes is equal to

$$
\begin{aligned}
N & =\sum_{j_{1}, \ldots, j_{d-1} \geq 0}\left\lfloor\frac{n}{p_{1} \prod_{0<k<d} p_{k}^{j_{k}}}\right\rfloor \prod_{0<k<d} p_{k}^{j_{k}} \\
& \geq \sum_{p_{1}^{j_{1}} \times \cdots \times p_{d-1}^{j_{d-1} \leq n / 2 p_{1}}}\left(\frac{n}{p_{1}}-\prod_{0<k<d} p_{k}^{j_{k}}\right) \\
& \geq \sum_{0 \leq m \leq \log n / \log p_{d-1}-2} \sum_{j_{1}+\cdots+j_{d-1}=m} \frac{n}{2 p_{1}} .
\end{aligned}
$$

It follows that

$$
N \gg \frac{n}{p_{1}}\left(\frac{\log n}{\log p_{d-1}}\right)^{d-1} .
$$

By choosing $p_{1}$ to be around $(\log n)^{d-1}$, we can thus find $n$ boxes that define a set system whose incidence matrix $A$ has at least $n\left(\log n / \log p_{d-1}\right)^{d-1}$ ones (note that we may have to pad with rows of zeros). We now show that $A$ is square-free (i.e., has no $2 \times 2$ submatrix of ones).

Consider the intersection of the special box $B$ with another special box $B^{\prime}$ with parameters $\left(j_{1}^{\prime}, \ldots, j_{d-1}^{\prime}\right)$. Without loss of generality, assume that $Q<Q^{\prime}$. (Note that the case $Q=Q^{\prime}$ corresponds to an empty intersection.) The intersection of two intervals of type $(k, j)$ and $\left(k, j^{\prime}\right), j^{\prime}<j$, is either empty or an interval of type $(k, j)$. This implies that $B \cap B^{\prime}$ is a box $J_{1} \times \cdots \times J_{d}$, where $J_{k}(k<d)$ is an interval of type $\left(k, \max \left\{j_{k}, j_{k}^{\prime}\right\}\right)$, and $J_{d}$ has length at most $p_{1} Q / n$. Assume that the box $B \cap B^{\prime}$ contains a point of $P$ and let $m$ be its index. By the Chinese Remainder Theorem, $m$ is completely specified modulo $\prod p_{k}^{\max \left\{j_{k}, j_{k}^{\prime}\right\}}$, and hence modulo $p_{i} Q$, for some $0<i<d$. We know that the 
point's $d$ th coordinate $m / n$ lies in an interval $J_{d}$ of length at most $p_{1} Q / n$, therefore $m$ is uniquely determined. Thus, two special boxes intersect in at most one point of $P$. It follows that $A$ is square-free, and by Lemma 3.3, the proof of Theorem 3.1 is complete.

Proof of Theorem 3.2. We exhibit a set $P$ of $n$ points in $\mathbf{R}^{d}$ along with a collection $\left\{S_{q}\right\}$ of $n$ slabs, such that: (i) each slab contains roughly $n^{1-2 /(d+1)}$ points, and (ii) the intersection of any $k \geq \log n$ slabs contains at most a logarithmic number of points. The proof technique is similar to [7]; there are some differences, however, so we provide the details below.

Let $H_{q}$ be the hyperplane of equation $\langle p, q\rangle-\|q\|_{2}^{2}=0$ : this is the hyperplane normal to $O_{q}$ passing through $q$. Fix a parameter $w$; we let $S_{q}$ denote the slab of width $w$ consisting of all the points at most $w / 2$ away from $H_{q}$. To specify the collection of slabs $\left\{S_{q}\right\}$, it thus suffices to provide a set $Q$ of $n$ points $q$. First, we show that if any $d$ of the points of $Q$ are sufficiently spread apart (in a sense to be formalized below), then the corresponding slabs have a small intersection. Next, by appealing to the results on Heilbronn's problem of [3], we are able to exhibit a suitable set $Q$. Finally, throwing in a set of random points in the unit cube provides $P$ and completes the construction of the set system. We now give the details of the construction of $Q$. (We use the notation $\operatorname{vol}_{d}(A)$ and $\operatorname{conv}(A)$ to refer to the $d$-dimensional volume and the convex hull of $A$, respectively.)

Lemma 3.4. Let $q_{1}, \ldots, q_{d}$ be $d$ points in $[0,1]^{d}$, and assume that the central projection $q_{i}^{\prime}$ of each $q_{i}$ on the hyperplane $x_{1}=1$ also lies in $[0,1]^{d}$. Then

$$
\operatorname{vol}_{d} \bigcap_{i=1}^{d} S_{q_{i}} \ll \frac{w^{d}}{\operatorname{vol}_{d-1}\left(\operatorname{conv}\left(q_{1}^{\prime}, \ldots, q_{d}^{\prime}\right\}\right)} .
$$

Proof. Let $\left[u_{1}, \ldots, u_{d}\right]$ denote the matrix whose columns are the vectors $u_{i}$ spanning the parallelepiped $\bigcap S_{q_{i}}$. Note that each $u_{i}$ has the direction specified by the intersection of hyperplanes bounding the slabs $S_{q_{j}}$, for all $j \neq i$. We easily derive

$$
\operatorname{det}\left(\left[u_{1}, \ldots, u_{d}\right]^{\top}\left[q_{1}, \ldots, q_{d}\right]\right)=w^{d} \prod_{i}\left\|q_{i}\right\|_{2},
$$

and therefore

$$
\begin{aligned}
\operatorname{vol}_{d} \bigcap_{i=1}^{d} S_{q_{i}} & =w^{d} \frac{\prod_{i}\left\|q_{i}\right\|_{2}}{\left|\operatorname{det}\left[q_{1}, \ldots, q_{d}\right]\right|} \\
& =w^{d} \frac{\prod_{i}\left\|q_{i}^{\prime}\right\|_{2}}{\left|\operatorname{det}\left[q_{1}^{\prime}, \ldots, q_{d}^{\prime}\right]\right|} \\
& \ll \frac{w^{d}}{\left|\operatorname{det}\left[q_{1}^{\prime}, \ldots, q_{d}^{\prime}\right]\right|},
\end{aligned}
$$

from which the lemma easily follows. 
Choose an integer $m=\left\lfloor c_{0} n w\right\rfloor$, for some constant $c_{0}>0$. By Theorem 4.10 of [3], we can place $m$ points in $(1,0, \ldots, 0)+[0,1]^{d-1}$ so that the convex hull of any $k \geq \log m$ points has $(d-1)$-dimensional volume at least $\Omega(k / m)$. For each such point $q^{\prime}$, place points on the segment $O q^{\prime}$ at intervals of length $w$. This gives us $O\left(c_{0} n\right)$ points $q:$ if $w$ is small enough then, for at least a constant fraction of them, the slab $S_{q}$ intersects the cube $[0,1]^{d}$ in a polytope of volume $\Omega(w)$. By choosing $c_{0}$ large enough, we can find $n$ such points, which thus form the set $Q$. To summarize, the set $Q$ consists of $n$ points such that, for any $q \in Q$,

$$
\operatorname{vol}_{d} S_{q} \cap[0,1]^{d} \gg w .
$$

Also, for any distinct $q_{1}, \ldots, q_{k} \in Q$, with $k \geq \log n$, either at least two $q_{i}$ 's have the same central projection $q_{i}^{\prime}$, in which case $\operatorname{vol}_{d} \bigcap_{i=1}^{k} S_{q_{i}}=0$, or else

$$
\operatorname{vol}_{d-1}\left(\operatorname{conv}\left\{q_{1}^{\prime}, \ldots, q_{k}^{\prime}\right\}\right) \gg \frac{k}{m} \text {. }
$$

By triangulating the convex hull of $q_{1}^{\prime}, \ldots, q_{k}^{\prime}$, using $O\left(k^{\lfloor(d-1)\rfloor / 2}\right)$ simplices, we derive the existence of $d$ points, say, $q_{1}^{\prime}, \ldots, q_{d}^{\prime}$, whose convex hull has volume at least $\Omega\left(k^{2-\lceil d / 2\rceil} / m\right)$. By Lemma 3.4, this shows that in all cases

$$
\operatorname{vol}_{d} \bigcap_{i=1}^{k} S_{q_{i}} \leq \operatorname{vol}_{d} \bigcap_{i=1}^{d} S_{q_{i}} \ll n w^{d+1} k^{\lceil d / 2\rceil-2} .
$$

We define the point set $P$ by choosing $n$ points in $[0,1]^{d}$ at random uniformly and independently. Let $w=b n^{-2 /(d+1)}(\log n)^{c}$, for some fixed $b>0$ and $c=$ $(3-\lceil d / 2\rceil) /(d+1)$. Set $k=\lceil\log n\rceil$; we can ensure that $k$-wise intersection of slabs contain only $O(\log n)$ points. We use standard Chernoff bounds below; see, e.g., [1]. By (5) there exists a constant $c_{1}>0$ such that the probability that $\bigcap_{i=1}^{k} S_{q_{i}}$ contains more than $c_{1} n^{2} w^{d+1} k^{\lceil d / 2\rceil-2}=\Theta(\log n)$ points is less than $n^{-d}$. Thus, with probability greater than $1-\left(\begin{array}{l}n \\ k\end{array}\right) n^{-d}$, no $k$-wise intersection of slabs contains more than $O(\log n)$ points. Note that by (5) the factor of $\left(\begin{array}{l}n \\ k\end{array}\right)$ can be replaced by $\left(\begin{array}{l}n \\ d\end{array}\right)$, so the probability is actually greater than $1 / 2$.

Similarly, by (4) the probability that a given $S_{q}$ contains fewer than $c_{2}$ wn points is less than $e^{-\Omega(w n)}<e^{-n^{1 / 4}}$, for some constant $c_{2}>0$. So, with probability greater than $1 / 2$, all the slabs $S_{q}$ contain $c_{2} w n$ points or more. We derive the existence of a set $P$ such that every slab $S_{q}(q \in Q)$ contains $\Omega(w n)$ points and no subset of $\lceil\log n\rceil$ slabs has an intersection containing more than $O(\log n)$ points.

The set $P$ and the slabs $S_{q}$ form a set system $A$ with no $p \times q$ submatrix of ones, where both $p$ and $q$ are in $O(\log n)$. It follows from Lemma 3.3 that the semigroup complexity of the map $x \mapsto A x$ is $\Omega\left(w n^{2} / \log ^{2} n\right)$. Since the constant $c$ is at least $-1 / 2$, this bound is at least $\Omega\left(n^{2-2 /(d+1)}(\log n)^{-5 / 2}\right)$, which proves Theorem 3.2. Note that the polylogarithmic factor can be tightened a little by keeping the exact value of $c$.

\section{Concluding Remarks}

One of the most intriguing open problems is, of course, to improve the $\Omega(n \log \log n)$ lower bound, or to extend it to rings or fields. Also, the discrepancy theory literature is vast 
and rich in powerful mathematical techniques. To establish further links to complexity theory would be very interesting.

\section{Acknowledgments}

I wish to thank the referee for several helpful comments.

\section{References}

1. Alon, N., Spencer, J. The Probabilistic Method, Wiley, New York, 1992.

2. Beck, J., Chen, W. W. L. Irregularities of Distribution, Cambridge Tracts in Mathematics, vol. 89, Cambridge University Press, Cambridge, 1987.

3. Chazelle, B. Lower bounds on the complexity of polytope range searching, J.Amer. Math. Soc., 2 (1989), 637-666.

4. Chazelle, B. Lower bounds for orthogonal range searching: II. The arithmetic model, J. Assoc. Comput. Mach., 37 (1990), 439-463.

5. Chazelle, B. Computational geometry: A retrospective, in: Computing in Euclidean Geometry, 2nd edn., eds. D.-Z. Du and F. Hwang, World Scientific Press, Singapore, 1995, pp. 22-46.

6. Chazelle, B. A spectral approach to lower bounds with applications to geometric searching, SIAM J. Comput. (1996), to appear.

7. Chazelle, B., Rosenberg, B. Lower bounds on the complexity of simplex range reporting on a pointer machine, Comput. Geom. Theory Appl. 5 (1996), 237-247. Preliminary version in Proc. 19th ICALP, LNCS 623, Springer-Verlag, Berlin, 1992, pp. 439-449.

8. Edelsbrunner, H. Algorithms in Combinatorial Geometry, Springer-Verlag, New York, 1987.

9. Erickson, J. New lower bounds for Hoperoft's problem, Proc. 11th Ann. ACM Symp. on Computational Geometry, 1995, to appear.

10. Fredman, M. L. A lower bound on the complexity of orthogonal range queries, J. Assoc. Comput. Mach., 28 (1981), 696-705.

11. Fredman, M. L. Lower bounds on the complexity of some optimal data structures, SIAM J. Comput., 10 (1981), 1-10.

12. Lancaster, P., Tismenetsky, M. The Theory of Matrices, 2nd edn., Academic Press, New York, 1985.

13. Matoušek, J. Geometric range searching, Tech. Report B-93-09, Free University Berlin, 1993.

14. Mehlhorn, K. Data Structures and Algorithms 3: Multidimensional Searching and Computational Geometry, Springer-Verlag, Heidelberg, 1984.

15. Morgenstern, J. Note on a lower bound of the linear complexity of the fast Fourier transform, J. Assoc. Comput. Mach., 20 (1973), 305-306.

16. Mulmuley, K. Computational Geometry: An Introduction Through Randomized Algorithms, Prentice-Hall, Englewood Cliffs, NJ, 1994.

17. Roth, K. F. On irregularities of distribution, Mathematika, 1 (1954), 73-39.

18. Yao, A. C. On the complexity of maintaining partial sums, SIAM J. Comput., 14 (1985), 277-288.

19. Yao, F. F. Computational geometry, in: Algorithms and Complexity, Handbook of Theoretical Computer Science, Vol. A, ed. J. van Leeuwen, Elsevier, Amsterdam, 1990, pp. 343-389.

Received December 21, 1994, and in revised form March 11, 1996. 